\title{
GLACIER SLIDING DOWN AN INCLINED WAVY BED
}

\author{
By L. W. MORLAND
}

(School of Mathematics and Physics, University of East Anglia, Norwich NR4 7TJ, England)

\begin{abstract}
The treatments by Nye and Kamb of glacier sliding over a wavy bed with small slope, which assume the ice to be approximated by a Newtonian fluid of high viscosity, are complemented by the inclusion of the glacier depth and the inclination of the bed to the horizontal. The driving force of the motion, gravity, is therefore present in the flow equations and defines immediately the mean drag on the bed. A geothermal heat flux is also included in order to estimate its possible effect on the flow. A complex variable method is used to determine the velocity and temperature fields to second order in the bed slope. These fields satisfy the zero shear traction and pressure-melting-regelation conditions to the same order on the actual bed profile. It is the balance of the second-order term which determines explicitly the (zero order) basal-sliding velocity and surface velocity in terms of the geometry and physical properties of both ice and bed. An explicit solution is illustrated for a sinusoidal bed, and a simple criterion for the onset of cavitation is obtained.

RÉsumé. Glissement d'un glacier sur un lit incliné ondulé. Les théories de Nye et Kamb pour le glissement d'un glacier sur un lit ondulé à faible pente, assimilant la glace à un fluide newtonien à haute viscosité, sont complétées par la prise en compte de l'épaisseur du glacier et de l'inclinaison du lit sur l'horizontale. La force qui provoque le mouvement, la gravité, est donc présente dans les équations de l'écoulement et définit immédiatement le frottement moyen sur le lit. On introduit également un flux de chaleur géothermique pour estimer son effet possible sur l'écoulement. Une méthode à variable complexe est utilisée pour déterminer les champs de vitesse et de température jusqu'au second ordre dans la pente du lit, qui satisfassent les conditions d'un cisaillement nul et de pression-fusion-congélation jusqu'à cet ordre sur le profil réel du lit. C'est le terme du second ordre qui détermine explicitement le glissement au fond (voisin de zéro), la vitesse et la vitesse en surface d'après les propositions géométriques et physiques du lit et de la glace. Une solution explicite est donnée en exemple pour un lit sinusoïdal et on obtient un critère simple pour le déclenchement de la cavitation.
\end{abstract}

Zusammenfassung. Gletschergleiten über ein geneigtes, gewelltes Bett. Nye's und Kamb's Behandlung des Gletschergleitens über ein gewelltes Bett geringer Neigung unter der Annahme, das Eis sei annähernd eine Newtonsche Flüssigkeit hoher Viskosität, wird durch die Einbeziehung der Eisdicke und der Neigung des Bettes gegen die Horizontale ergänzt. Die Schwerkraft als Triebkraft der Bewegung geht daher in die Fliessgleichungen ein und bestimmt unmittelbar den mittleren Schub auf das Bett. Weiter wird ein geothermischer Wärmefluss eingeführt, um seinen möglichen Einfluss auf das Fliessen abschätzen zu können. Zur Bestimmung der Geschwindigkeits- und Temperaturfelder bis zur 2 Ordnung der Bettneigung, welche die Bedingungen für das Verschwinden des Scherzuges und für Regelation unter Druckschmelze bis zu dieser Ordnung auf dem tatsächlichen Bettprofil erfüllen, wird eine komplexe Variationsmethode herangezogen. Die Ausgewögenheit der Glieder 2 Ordnung ist es, die explizit die basale Gleitgeschwindigkeit (nullter Ordnung) und die.Oberflächengeschwindigkeit als Funktion der geometrischen und physikalischen Eigenschaften von Eis und Bett bestimmt. Eine explizite Lösung qird für ein sinusförmiges Bett vorgelegt; dabei ergibt sich ein einfaches Kriterium für das Einsetzen der Kavitation.

\section{INTRODUGTION}

The overall motion of a temperate glacier consists of a relative deformation or flow through the ice mass together with basal sliding over the bed (which may make a significant contribution to the observed surface velocity). The internal flow is governed by the constitutive response of the ice whereas basal sliding depends on conditions at the ice-bed interface. Specifically it is the presence of a thin water layer, caused by pressure melting and refreezing as the ice flows over undulations, which "lubricates" the bed and allows slip to occur.

The regelation mechanism was proposed first by Weertman (1957) who modelled the bed as a regular array of rectangular obstacles. The latent heat released by freezing on the lowpressure down-stream face is conducted through the obstacle to melt the ice on the highpressure up-stream face. An energy argument and an assumed value for the shear stress at the bed then lead to a basal-sliding velocity due to pressure-melting which is expressed in terms of the obstacle and spacing lengths. An estimate of the creep-rate is made by using Glen's law for the ice response and by making further assumptions about the stresses on the obstacle. Next, an expression for the sliding velocity due to a creep process is inferred. 
The two estimates of sliding velocity, one based on regelation and the other on creep, decrease and increase with the length scale of the obstacle, respectively. Weertman proposes, therefore, that since all length scales will exist in practice, sliding is controlled by the length giving the same velocity for both mechanisms. Equating the two expressions gives the sliding velocity in terms of the drag and a roughness parameter - a ratio of obstacle length to spacing. The theory is extended in a similar manner (Weertman, 1964) to account for cavitation on the down-stream faces. Later, Weertman (I97I) defends this simple "semi-quantitative" theory against the criticisms of Nye (1969, 1970) and Kamb (1970). However, the theory of Weertman relies crucially on estimates of stress and a sliding velocity deduced from an inferred mean creep rate. There is also the difficulty of relating obstacle size and spacing to a more general bed profile; further, there is no demonstration that a flow field with the required features exists. A more elaborate treatment in the same spirit is proposed by Lliboutry (1968) who considers a sinusoidal bed profile, accounts for cavitation, and discusses various basal friction laws, in other words, the relationships between the mean drag, the normal pressure, and the sliding velocity.

Nye (1969, 1970) and Kamb (1970) focus upon the need for an exact flow and heat conduction solution which satisfies the regelation conditions at the ice-bed interface. For this purpose Nye assumes that the ice response can be approximated as a Newtonian (incompressible) fluid of high viscosity and that the bed profile is periodic with small slope. Because of this the Reynolds number is very low, inertia terms are negligible, and slow steady viscous flow is assumed. Further, the water layer thickness is negligible compared with the length scale of the undulations, so the layer is treated as a surface distribution of heat sources (sinks) which coincide with the bed surface and which give rise to the latent heat of freezing (melting). The interface conditions are linearized to define a half-space problem for the ice flow after ignoring the upper glacier surface and introducing a small slope parameter $\epsilon$. This problem is treated by Fourier analysis, first for a plane flow and then for three-dimensional flow. Second-order expressions in $\epsilon$ are obtained for the flow field over a sinusoidal bed. Expressions for the mean drag are deduced and the results are extended by the statistical analysis of a general bed profile. The solution assumes that no cavitation occurs. Kamb (1970) obtains the same linear solution, demonstrating that the ice motion may be neglected in the heat conduction, but uses this as a starting point for an approximate treatment when the ice satisfies the more realistic non-linear Glen law.

The present paper complements the solution of Nye and Kamb for Newtonian plane flow (small bed slope) by incorporating explicitly the depth $h$ of the glacier and the inclination $\alpha$ of a (mean) bed line to the horizontal, both parameters assumed uniform over the length scale of interest. Thus, gravity (which is the driving force for the glacier motion) is included in the balance equations for slow viscous flow, and a flow solution is determined without the need to introduce an artificial shear stress at some distance from the bed (Nye, i 969 ). Furthermore, the solution satisfies an upper-surface condition of normal atmospheric pressure $p_{\mathrm{a}}$ within the approximate expansion scheme. It is supposed that there is no net longitudinal stress gradient, only a periodic variation induced by the bed undulation, and that the flow is steady, that is, no rigid body acceleration of order $g \sin \alpha$ parallel to the bed occurs; this is consistent with normal flow conditions. The mean drag on the bed is therefore

$$
\tau=\rho g h \sin \alpha,
$$

where $\rho$ is the ice density.

Since shear stress in a thin water layer at the bed is negligible compared with $\tau$, one boundary condition on the bed is that of zero tangential traction. This is an idealized condition which assumes that neither draining nor pinching-out of the water layer occur, and that there is no cavitation so that the ice boundary is everywhere the bed surface. The drag is the longitudinal resultant of the pressure acting over the undulations. The other bed 
conditions link the ice flow and heat conduction through the pressure-melting and regelation relations. For completeness a uniform geothermal heat flux acting normal to the bed is included at large distances from the bed, so that its effect on the flow can be estimated for levels which are observed typically. The effect is shown to be small. The flow solution determines explicitly the basal sliding velocity $U_{\mathrm{b}}$, and surface velocity $U_{\mathrm{s}}$, in terms of $h, \alpha$, and the bed geometry for given ice and bed properties. An illustration for a sinusoidal bed profile shows that $U_{\mathrm{b}}$ is very sensitive to the wavelength of the undulation. The variations of $U_{\mathrm{b}}$ with seasonal changes of melt water (Lliboutry, 1968) cannot be described by this model of the bed conditions.

The small parameter $\epsilon \ll I$ is chosen as the maximum bed slope (relative to the inclined bed line), and for mathematical completeness the bed profile is extended periodically to infinity. Dimensionless coordinates are introduced through a length scale $\lambda$ defined so as to make the maximum amplitude of the dimensionless bed profile unity from a given bed line. The profile is assumed to be sufficiently differentiable for expansion procedures to a required order in $\epsilon$. The coefficients of leading terms are restricted to order unity by this choice of coordinates. It is assumed that $\lambda / h$ is sufficiently small for the upper surface conditions to be satisfied to the required order in $\epsilon$. In fact, the perturbation in the flow from a basic laminar flow is found to decay exponentially with height so that this is not a strong restriction. The approach here is to seek half-plane solutions to the perturbation flow equations in the ice and the heat conduction equations in the ice and bed which satisfy, to the required order in $\epsilon$, the boundary conditions on the actual bed surface. That is, the bed surface conditions are not applied on the boundary of a half-plane as in the earlier solutions, which can therefore be correct only in the leading term of the expansions.

A complex variable method is used and solutions to order $\epsilon^{2}$ obtained explicitly, though the expansions given determine valid solutions to order $\epsilon^{3}$ with a further iteration. The evaluations are elementary for a profile which can be adequately modelled by a low-order truncated Fourier series, and the solution is illustrated for a sinusoidal bed. A simple cavitation criterion is found which depends only on $x$ and $\epsilon$. To an order of the first power of $\epsilon$, the velocity-field

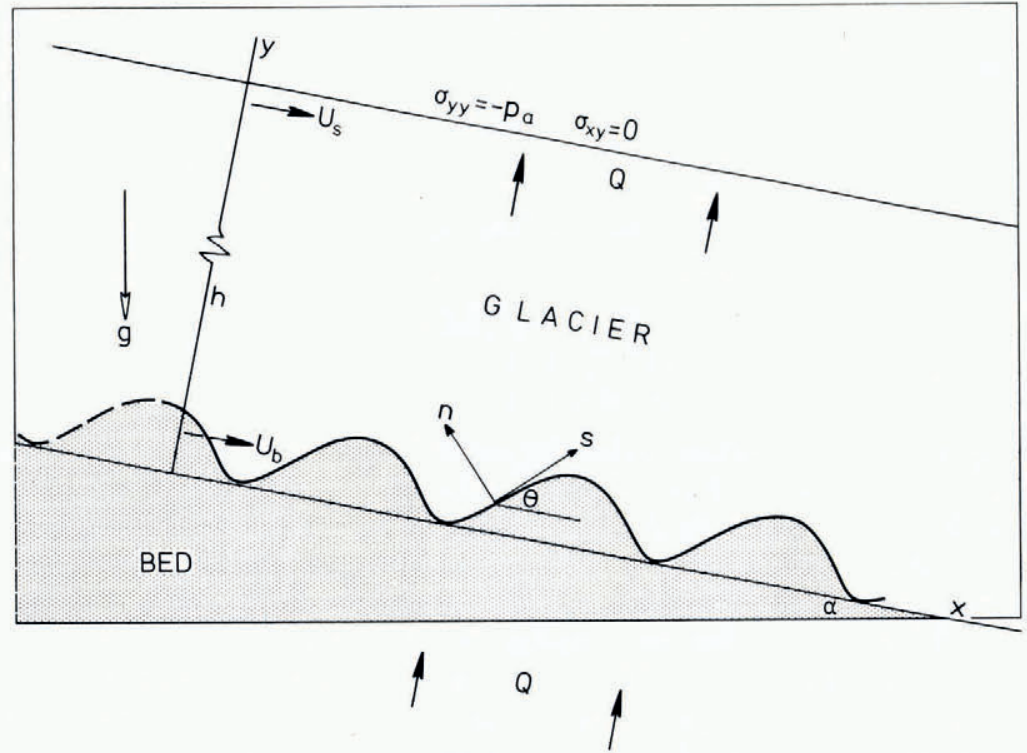

Fig. 1. Glacier flow over a wary bed. 
perturbation agrees with the solution of Nye (1969). However, whereas the present $\epsilon^{2}$ terms are bounded, Nye has an unbounded term due to the artificial shear stress at infinity which is introduced to drive the motion. The determination of a parameter to order one, which in turn determines the basal sliding velocity, occurs in the balance of order $\epsilon^{2}$ terms, so it is essential to construct a consistent expansion scheme to order $\epsilon^{2}$. Furthermore, with classical boundary conditions of zero velocity there are no bounded half-plane solutions for flow which are non-trivial (Langlois, I964), so the present solution verifies that the present boundary conditions define a well-posed flow problem.

\section{BED PROFILE AND BOUNDARY CONDITIONS}

Figure I displays the idealized flow problem. Coordinates $(x, y)$ are chosen along a bed line and normal to the bed respectively, with the $x$-axis inclined at angle $\alpha$ to the horizontal. The glacier flows in the $x$-direction, $U_{\mathrm{s}}$ is the surface velocity at $y=h$, and $U_{\mathrm{b}}$ is the basal sliding velocity defined as the $x$-velocity in a flow continued onto $y=0$. The bed profile is given by

$$
y_{\mathrm{b}}=f(x),
$$

where $f(x)$ is smooth, sufficiently differentiable for subsequent expansions, and supposed to extend periodically as $x \rightarrow \pm \infty$. This allows a well-posed mathematical problem; a different profile distant from the region of interest will not affect the local flow field. If $f(x)$ can be described adequately by a low-order truncated Fourier series the later solution is easily evaluated. Following Nye (1969, 1970) and Kamb (1970) it is assumed that the bed slope is everywhere small; thus,

$$
\epsilon=\left|f^{\prime}(x)\right|_{\max } \ll \mathrm{I} .
$$

Now we introduce the dimensionless coordinates $(X, Y)$ with a length scale $\lambda$ :

$$
\left.\begin{array}{l}
x=\lambda X \\
y=\lambda Y
\end{array}\right\}
$$

in which the bed profile becomes

$$
Y_{\mathrm{b}}=f(\lambda X) / \lambda=\epsilon F(X) .
$$

We then choose

$$
\lambda=f_{\mathrm{m}} / \epsilon,
$$

where

$$
f_{\mathrm{m}}=|f(x)|_{\max }
$$

Thus

$$
\left.\begin{array}{rl}
F(X) & =f(\lambda X) / f_{\mathrm{m}}, \\
|F(X)| & \leqslant \mathrm{r}, \\
\left|F^{\prime}(X)\right| & \leqslant \mathrm{r} .
\end{array}\right\}
$$

That is, the length scale $\lambda$ is defined so that the contributions of $F(X)$ and $F^{\prime}(X)$ to the expansion coefficients are necessarily of order unity. The only restriction on the amplitude $f_{\mathrm{m}}$ is Equation (6) with the strong inequality represented by Equation (3). My approach is to seek flow solutions in the half-plane $y>0(Y>0)$ which give correct values on the surface $r=Y_{\mathrm{b}}$, to a required order in $\epsilon$. Strictly, $Y_{\mathrm{b}}$ should lie inside the half-plane $Y_{\mathrm{b}} \geqslant \mathrm{o}$ as shown, but half-plane heat conduction solutions for the bed must be evaluated on $Y_{\mathrm{b}}$, and hence continued outside their domain if $r_{\mathrm{b}}>0$. The smoothness conditions on $f(x)$ required for the expansion procedure allow such extensions, so the bed line $y=0$ can be set anywhere in the vicinity of the bed surface. However, changing the bed line $y=0$ for a given profile shape changes $f_{\mathrm{m}}$ and the scale length $\lambda$, so that it must be confirmed that this scale change 
leaves the physical solution invariant. The change is equivalent to a uniform shift of the profile in the $y$-direction (either direction), so consider

$$
\begin{gathered}
y_{\mathrm{b}}=f(x)+c f_{\mathrm{m}} \quad(c=O(\mathbf{I})), \\
\frac{\lambda_{c}}{\lambda}=\left|\frac{f(x)}{f_{\mathrm{m}}}+c\right|_{\max }=\frac{\bar{c}}{c}=O(\mathrm{I}), \\
F_{c}(X)=\lambda / \lambda_{c} F\left(\lambda X / \lambda_{c}\right)+c / \bar{c} .
\end{gathered}
$$

Thus

$$
F_{c}^{\prime}(X)=F^{\prime}\left(\lambda_{c} X / \lambda\right) \quad F_{c}^{\prime \prime}(X)=\frac{\lambda_{c}}{\lambda} F^{\prime \prime}\left(\lambda_{c} X / \lambda\right) \ldots,
$$

and if $w(x)$ is any physical variable represented by

$$
W(X)=w(\lambda X) \quad W_{c}(X)=w\left(\lambda_{c} X\right),
$$

on the two scales, then

$$
W_{c}(X)=W\left(\lambda_{c} X / \lambda\right) \quad W_{c}^{\prime}(X)=\left(\lambda_{c} / \lambda\right) W^{\prime}\left(\lambda_{c} X / \lambda\right) .
$$

It will be seen that the subsequent solution is invariant under the transformations shown in Equations (I I) and (I3), and, by Equation (9), any order of magnitude statement concerning $\lambda$ applies also to $\lambda_{c}$.

An alternative coordinate transformation

$$
\bar{X}=X \quad \bar{T}=r-r_{\mathbf{b}}(X),
$$

proposed by Nye (1970) to make the bed surface $r \equiv$ o requires

and

$$
\frac{\partial}{\partial r} \rightarrow \frac{\partial}{\partial \bar{Y}}
$$

$$
\left.\frac{\partial}{\partial X} \rightarrow \frac{\partial}{\partial \bar{X}}-\Upsilon_{\mathrm{b}}{ }^{\prime}(X) \frac{\partial}{\partial \bar{Y}}=\frac{\partial}{\partial \bar{X}}+O(\epsilon) \frac{\partial}{\partial \bar{Y}},\right\}
$$

so continuing beyond the leading terms gives differential equations of a more complicated form and the biharmonic feature of the theory is lost. Expansion about $Y=0$ of solutions of the original equations is therefore the more appropriate approach.

It is assumed that the regelation process takes place everywhere on the surface producing a continuous thin water layer. The possible shear stress in such a water layer is negligible compared with $\tau$ so that one boundary condition for the ice at the bed surface is

$$
r=r_{\mathrm{b}}: \quad t_{s}=\mathrm{o},
$$

where $(s, n)$ denotes the local tangent and normal (inward to the ice) coordinates at a point on the bed surface, with the $s$-tangent direction inclined at angle $\theta$ to the bed line $y=0$. The condition represented by Equation $(16)$ is an idealization which requires that the water layer is nowhere "pinched-out" or drained away, and which assumes that the ice surface remains in contact with the surface layer; that is, no appreciable cavitation occurs. In terms of the stress tensor $\sigma$,

$$
r=Y_{\mathrm{b}}: \quad t_{s}=\cos 2 \theta \sigma_{x y}+\frac{1}{2} \sin 2 \theta\left(\sigma_{y y}-\sigma_{x x}\right)
$$

where

$$
\tan \theta=f^{\prime}(x)=\epsilon F^{\prime}(X) .
$$

The bed surface is everywhere at the pressure-melting point. Let $p_{0}$ be a mean pressure level for the bed, and $\left(p_{0}, T_{0}\right)$ a pressure-melting point. Then, for temperatures $T$ close to $T_{0}$ there is a linear relation between temperature and pressure-melting point for ice

$$
T-T_{0}=-C\left(p-p_{0}\right)
$$


where $C=0.7 \times 10^{-7}{ }^{\circ} \mathrm{C} \mathrm{m}^{2} \mathrm{~N}^{-1}$ (Nye, I 969 ). Kamb (1970) points out that the hydrostatic pressure $p$ is the correct stress in Equation (I9). Thus, if $T$ is the temperature in the ice and $S$ the temperature in the bed, temperature continuity at the bed surface gives

$$
\Upsilon=\Upsilon_{\mathrm{b}}: \quad S-T_{0}=T-T_{0}=-C\left(p-p_{0}\right) .
$$

If $V_{\mathrm{n}}$ is the normal component of ice velocity on the bed surface (directed into the ice so that a positive value denotes local freezing and a negative value indicates melting), then there is a surface distribution of heat sources with density per unit length of bed $L V_{\mathrm{n}}$, unit length normal to the plane, where $L$ is the latent heat (Nye, I 969 ) and $L=2.8 \times \mathrm{IO}^{8} \mathrm{~J} \mathrm{~m}^{-3}$. Let $\mathcal{N}=n / \lambda$ be the normal coordinate in $(X, Y)$ coordinates, then the ice and bed temperature fields satisfy the surface flux condition

$$
r=r_{\mathrm{b}}: \quad-k_{\mathrm{i}} \frac{\partial T}{\partial \mathcal{N}}+k_{\mathrm{b}} \frac{\partial S}{\partial \mathcal{N}}=\lambda L V_{\mathrm{n}},
$$

where $k_{\mathrm{i}}, k_{\mathrm{b}}$ are the thermal conductivities of the ice and bed respectively. $k_{\mathrm{i}}=2.0$ $\mathrm{J} \mathrm{m}^{-1} \mathrm{~s}^{-1}{ }^{\circ} \mathrm{C}^{-1}$ and $k_{\mathrm{b}}=r k_{\mathrm{i}}$ where $r$ has a range from $\mathrm{I}$ to 2 for typical bed rocks; the value $r=\mathrm{r} .6$ appropriate to granite is used in a later calculation.

The stress in the ice is given in terms of the two velocity components $V_{x}, V_{y}$, by the constitutive law, so Equations (I6), (20) and (2I) are four bed surface conditions for $V_{x}, V_{y}$, $T, S$. On the glacier surface

$$
r=h / \lambda: \quad \sigma_{y y}=-p_{\mathrm{a}}, \quad \sigma_{x y}=0,
$$

where $p_{\mathrm{a}}$ is atmospheric pressure. Strictly, the free-surface condition (Equation (22)) is compatible with the restriction $y \equiv h$ only if $V_{y} \equiv 0$ there, but it will be shown that this is valid to any order in $\epsilon$ under the weak restriction

$$
\lambda / h=O(\mathrm{I}),
$$

since the solution gives exponential decay in $Y$ for $V_{y}$. The flow is represented as the sum of a laminar flow satisfying Equation (22) exactly, but not Equation (I6), and a perturbation which is not assumed to be small near the bed, only at $y=h$, so that Equation (22) is satisfied to the required order. The perturbation is constructed as a half-plane solution and Equation (23) is the requirement. Similarly, the ice and bed are treated as half-planes for the heat conduction solutions satisfying Equations (20) and (2I) on the bed surface. A geothermal heat flux $Q$ normal to the bed is included by the conditions

$$
\left.\begin{array}{l}
T \approx-\frac{\lambda Q}{k_{\mathrm{i}}} r \quad \text { as } r \rightarrow \infty, \\
S \approx-\frac{\lambda Q}{k_{\mathrm{b}}} r \quad \text { as } r \rightarrow-\infty .
\end{array}\right\}
$$

A typical value for $Q$ of $4 \times \mathrm{IO}^{-2} \mathrm{~J} \mathrm{~m}^{-2} \mathrm{~s}^{-1}$ (Paterson, $\mathrm{I} 969$ ) is used for later estimates.

\section{Flow equations}

The ice is assumed to be an incompressible Newtonian fluid of high viscosity $\mu$. A value for $\mu$ of $3 \times 10^{12} \mathrm{~N} \mathrm{~m}^{-2} \mathrm{~s}$ (Nye, 1969) is used in the calculations. Thus

$$
\left.\begin{array}{c}
\frac{\partial V_{x}}{\partial x}+\frac{\partial V_{y}}{\partial y}=0 \\
\sigma_{x x}=-p+2 \mu \frac{\partial V_{x}}{\partial x} \quad \sigma_{y y}=-p+2 \mu \frac{\partial V_{y}}{\partial y} \quad \sigma_{x y}=\mu\left(\frac{\partial V_{x}}{\partial y}+\frac{\partial V_{y}}{\partial x}\right) .
\end{array}\right\}
$$


Momentum balance for slow viscous plane flow under gravity requires that

$$
\left.\begin{array}{c}
\frac{\partial \sigma_{x x}}{\partial x}+\frac{\hat{\partial} \sigma_{x y}}{\partial y}+\rho g \sin \alpha=0 \\
\frac{\partial \sigma_{x y}}{\partial x}+\frac{\partial \sigma_{y y}}{\partial y}-\rho g \cos \alpha=0 .
\end{array}\right\}
$$

Let

where

$$
V_{x} / U_{\mathrm{s}}=\mathrm{I}-\kappa(\mathrm{I}-\lambda Y / h)^{2}+u, \quad V_{y} / U_{\mathrm{s}}=v
$$

$$
\kappa=\frac{\rho g \sin \alpha h^{2}}{2 \mu U_{\mathrm{s}}},
$$

and also

$$
p=p_{0}-\rho g \cos \alpha \lambda \gamma+\frac{2 \mu U_{\mathrm{s}}}{\lambda} P
$$

where

$$
\left.\begin{array}{c}
p_{\mathrm{o}}=p_{\mathrm{a}}+\rho g \cos \alpha h, \\
T=T_{0}-\frac{\lambda Q}{k_{\mathrm{i}}} \Upsilon+\frac{2 \mu C U_{\mathrm{s}}}{\lambda} \tilde{T}, \\
S=T_{0}-\frac{\lambda Q}{k_{\mathrm{b}}} r+\frac{2 \mu C U_{\mathrm{s}}}{\lambda} \tilde{S} .
\end{array}\right\}
$$

In these equations $u$ and $v$ are dimensionless velocity components (with unit $U_{\mathrm{s}}$ ) while $P$ is a dimensionless pressure (with unit $2 \mu U_{\mathrm{s}} / \lambda$ ) superposed on a laminar flow which balances the body force (gravity) and which satisfies the upper surface condition (Equation (22)), $p_{0}$ is the overburden pressure on a bed line $Y=0$ in the laminar flow. $\tilde{T}$ and $\tilde{S}$ are dimensionless temperatures (with unit $2 \mu C U_{\mathrm{s}} / \lambda$ ) in the ice and bed superposed on a uniform flux field satisfying Equation (24). They satisfy the steady heat conduction equations in their respective domains

$$
\nabla^{2} \tilde{T}=0, \quad \nabla^{2} \tilde{S}=0 .
$$

Here the ice motion is neglected (Kamb, 1970) and $\nabla^{2}$ is the two-dimensional Laplacian in $(X, Y)$ coordinates. Thus, absorbing constants into $T_{0}$ :

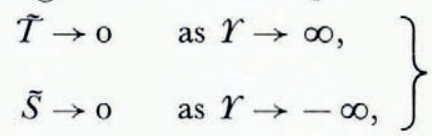

and Equation (20) can be written as the two relations

$$
\begin{aligned}
r & =r_{\mathrm{b}}: & \tilde{T} & =-P+B r_{\mathrm{b}}, \\
r & =r_{\mathrm{b}}: & \tilde{T}-\tilde{S} & =D \Upsilon_{\mathrm{b}},
\end{aligned}
$$

where

and

$$
\left.\begin{array}{l}
B=\frac{\kappa}{\sin \alpha}\left(\cos \alpha+\frac{Q}{\rho g k_{\mathrm{i}} C}\right)\left(\frac{\lambda}{h}\right)^{2} \\
D=\frac{r-\mathrm{I}}{r} \frac{\kappa}{\sin \alpha} \frac{Q}{\rho g k_{\mathrm{i}} C}\left(\frac{\lambda}{h}\right)^{2}
\end{array}\right\}
$$

$D=\mathrm{o}$ if $Q=\mathrm{o}$ or if the two conductivities are equal, that is, $r=k_{\mathrm{b}} / k_{\mathrm{i}}=\mathrm{I}$. With the values 
given earlier, $Q /\left(\rho g k_{\mathrm{i}} C\right)=31.66$, and to a good approximation the $\cos \alpha$ term in $B$ can be neglected and

$$
D \approx \frac{r-\mathrm{I}}{r} B, \quad B \approx \frac{3^{2 \kappa}}{\sin \alpha}\left(\frac{\lambda}{h}\right)^{2} .
$$

This reduction is not appropriate if $Q$ is appreciably smaller than the adopted value, when the full expressions given by Equations (36) must be used. The flux condition, Equation (2I), becomes

$$
r=r_{\mathrm{b}}: \quad \frac{\partial \tilde{T}}{\partial \mathcal{N}}-r \frac{\partial \tilde{S}}{\partial \mathcal{N}}=-2\left(\frac{\lambda}{\lambda_{*}}\right)^{2} \frac{V_{\mathrm{n}}}{U_{\mathrm{s}}},
$$

where

$$
\lambda_{*}^{2}=\frac{4 \mu k_{\mathrm{i}} C}{L}=0.006 \mathrm{o} \mathrm{m}^{2}
$$

for the adopted values. A value for $\lambda_{*}$ of $0.077 \mathrm{~m}$ is equivalent to the Nye (1969) value for $k_{*}{ }^{-1}$ (in his notation). By Equation (27)

$$
r=r_{\mathrm{b}}: \quad \frac{V_{\mathrm{n}}}{U_{\mathrm{s}}}=v \cos \theta-\sin \theta\left\{\mathrm{I}-\kappa+2 \kappa \frac{\lambda}{h} r_{\mathrm{b}-\kappa}\left(\frac{\lambda}{h}\right)^{2} r_{\mathrm{b}^{2}}+u\right\} .
$$

The flow formulae (Equations (25)-(29)) reduce to

$$
\nabla^{2} P=0, \quad \frac{\partial P}{\partial X}=\frac{1}{2} \nabla^{2} u, \quad \frac{\partial P}{\partial Y}=\frac{1}{2} \nabla^{2} v,
$$

which are the equations for slow viscous plane flow for a velocity field $(u, v)$ and pressure $P$ in the absence of body force and in the case where the viscosity is 0.5 (Langlois, 1964). The dimensionless stress $\boldsymbol{\Sigma}$ with unit $2 \mu U_{\mathrm{s}} / \lambda$ is given by

$$
\left.\begin{array}{c}
\Sigma_{x x}=-\frac{\lambda}{2 \mu U_{\mathrm{s}}} p+\frac{\partial u}{\partial X}, \quad \Sigma_{y y}=-\frac{\lambda}{2 \mu U_{\mathrm{s}}} p+\frac{\partial v}{\partial Y}, \\
\Sigma_{x y}=\kappa\left(\frac{\lambda}{h}\right)-\kappa\left(\frac{\lambda}{h}\right)^{2} r+\frac{1}{2}\left(\frac{\partial u}{\partial X}+\frac{\partial v}{\partial Y}\right) .
\end{array}\right\}
$$

Thus there exist analytic functions $\phi(z), \psi(z)$ of the complex variable $z=X+\mathrm{i} Y$ in $h>r>r_{\mathrm{b}}$ such that

$$
\begin{aligned}
v-\mathrm{i} u & =\phi(z)+z \overline{\phi^{\prime}(z)}+\overline{\psi(z)}, \\
P & =2 \operatorname{Im}\left\{\phi^{\prime}(z)\right\}, \\
\Sigma_{y y}-\Sigma_{x x} & =-2 \operatorname{Im}\left\{\bar{z} \phi^{\prime \prime}(z)+\psi^{\prime}(z)\right\}, \\
\Sigma_{x y}=\kappa\left(\frac{\lambda}{h}\right)[\mathrm{I} & \left.-\left(\frac{\lambda}{h}\right) r\right]+\operatorname{Re}\left\{\bar{z} \phi^{\prime \prime}(z)+\psi^{\prime}(z)\right\} .
\end{aligned}
$$

Similarly, from Equation (32), there exist analytic functions $\chi_{\mathrm{i}}(z), \chi_{\mathrm{b}}(z)$ with

and

$$
\left.\begin{array}{ll}
r>Y_{\mathrm{b}}: & \tilde{T}=\operatorname{Re}\left\{\chi_{\mathrm{i}}(z)\right\}, \\
r<r_{\mathrm{b}}: & \tilde{S}=\operatorname{Re}\left\{\chi_{\mathrm{b}}(z)\right\} .
\end{array}\right\}
$$

\section{Flow Solution}

Now let $u, v, \tilde{T}$ extend smoothly in $r>0$ onto $r=0$ (if $r_{\mathrm{b}}>0$ anywhere) and to infinity, and let $\tilde{S}$ extend smoothly in $r<\mathrm{o}$ onto $r=\mathrm{o}$ and to infinity, so that $u, v, \tilde{T}, \tilde{S}$ and the stress components are half-plane fields given by the Equations $(43)-(47)$. Define boundary values on $r=0$ such that 


$$
\begin{aligned}
& \text { GLACIER FLOW DOWN A SLOPE } \\
& u(X, 0)=U(X) \\
& v(X, 0)=V(X) \text {, } \\
& \tilde{T}(X, 0)=\Theta(X) \text {, } \\
& \tilde{S}(X, 0)=\Omega(X) \text {. }
\end{aligned}
$$

Note that $U, V, \Theta, \Omega$ are not values of the field variables on the bed surface, but are introduced to allow simple Cauchy integral representations of $\phi, \psi, \chi_{\mathrm{i}}, \chi_{\mathrm{b}}$. It is assumed that they vanish or have sinusoidal behaviour as $X \rightarrow \pm \infty$. Also, we prescribe infinity conditions

and

$$
\left.\begin{array}{rrrr}
r>0: & \phi, \psi \text { and } \chi_{\mathrm{i}} \rightarrow 0 & \text { as } z \rightarrow \infty, \\
r<0: & \chi_{\mathrm{b}} \rightarrow 0 & \text { as } z \rightarrow \infty,
\end{array}\right\}
$$

to be consistent with Equation (33) and the requirement in Equation (22). Then, Equations (43) and (47), subject to Equations (48) and (49), give (Muskhelishvili, 1954):

$$
\begin{array}{ll}
\phi(z)=\frac{\mathrm{I}}{2 \pi \mathrm{i}} \int_{-\infty}^{\infty} \frac{V(t)-\mathrm{i} U(t)}{t-z} \mathrm{~d} t, . & r>0, \\
\psi(z)=\frac{\mathrm{I}}{2 \pi \mathrm{i}} \int_{-\infty}^{*} \frac{V(t)+\mathrm{i} U(t)}{t-z} \mathrm{~d} t-z \phi^{\prime}(z), & r>0, \\
\chi_{\mathrm{i}}(z)=\frac{\mathrm{I}}{\pi \mathrm{i}} \int_{-\infty}^{\infty} \frac{\Theta(t)}{t-z} \mathrm{~d} t, & r>0, \\
\chi_{\mathrm{b}}(z)=-\frac{\mathrm{I}}{\pi \mathrm{i}} \int_{-\infty}^{\infty} \frac{\Omega(t)}{t-z} \mathrm{~d} t, & r<0 .
\end{array}
$$

Once $U, V, \Theta, \Omega$ are determined, Equations (50)-(53) and (43)-(47) give the physical field variables.

The following results are used repeatedly (Muskhelishvili, r954). If

$$
\Phi(z)=\int_{-\infty}^{\infty} \frac{W(t)}{t-z} \mathrm{~d} t, \quad r>0 \text { or } r<0,
$$

and $W(t)$ is differentiable and bounded at infinity, then

$$
\Phi^{\prime}(z)=\int_{-\infty}^{\infty} \frac{W^{\prime}(t)}{t-z} \mathrm{~d} t, \quad r>0 \text { or } r<0,
$$

with extension to higher derivatives, and

$$
\frac{\partial \Phi}{\partial Y}=\mathrm{i} \Phi^{\prime}(z), \quad \frac{\hat{c}}{\partial \Upsilon}\{\overline{\Phi(z)}\}=-\mathrm{i} \overline{\Phi^{\prime}(z)} .
$$

If $W(t)$ is continuous and vanishes or behaves sinusoidally at infinity,

$$
\Phi(z) \rightarrow \int_{-\infty}^{\infty} \frac{W(t)}{t-X} \mathrm{~d} t \pm \pi \mathrm{i} W(X) \quad \text { as } Y \rightarrow\left\{\begin{array}{l}
\mathrm{o}^{+} \\
0_{-}-
\end{array}\right.
$$


where i denotes the Cauchy principal value. Also

$$
\frac{1}{\pi} \int_{-x}^{+} \frac{W(t)}{t-X} \mathrm{~d} t=H[W](X), \quad \frac{\mathrm{d} H[W]}{\mathrm{d} X}=H\left[W^{\prime \prime}\right],
$$

where $H\left[W^{\prime}\right]$ is the Hilbert transform of $W^{\prime}(t)$ (Erdélyi, 1954), with the inversion theorem

$$
H[H[W]]=-W .
$$

Note the particular results

$$
\begin{aligned}
& H[\mathrm{I}]=0, \quad H[\sin k t]=\cos k X, \quad H[\cos k t]=-\sin k X, \\
& \left.\begin{array}{l}
\frac{\mathrm{I}}{\pi} \int_{-\infty}^{t} \frac{\sin k t}{t-z} \mathrm{~d} t=\exp (\mathrm{i} k X-k r) \\
\frac{\mathrm{I}}{\pi} \int_{-x}^{\cos k t} \frac{\cos }{t-z} \mathrm{~d} t=\mathrm{i} \exp (\mathrm{i} k X-k X)
\end{array}\right\} k>0, r>0,
\end{aligned}
$$

which are the only evaluations required when $F(X)$ is a truncated Fourier series, and which show the exponential decay in $r$. The first expression of Equation (6o) does not have the required conditions at infinity to satisfy the inversion theorem (Equation (59)).

The bed surface conditions (Equations (16), (34), (35), and (38)) can now be expressed in terms of Equations (43)-(47) and (50)-(53); that is, in terms of $U, V, \Theta$ and $\Omega$. However, assuming sufficient smoothness, the value of each quantity on $r=Y_{\mathrm{b}}(X)$ can first be approximated by a truncated Taylor series in $Y$ about $Y=0$ to the required order in $\epsilon$ at each $X$, and then the formulae used to evaluate quantities on $r=0$. If we adopt this process, and omit the lengthy but straightforward algebra using Equations $(54)-\left(5^{8}\right)$, the bed surface conditions become

$$
\begin{aligned}
\kappa(\lambda / h)\left\{\mathrm{I}-(\lambda / h) \epsilon F-2 \epsilon^{2}\left(F^{\prime}\right)^{2}\right\}+H\left[U^{\prime}\right]-\epsilon\left\{F H\left[V^{\prime \prime}\right]+2\left(F U^{\prime}\right)^{\prime}\right\}+\epsilon^{2}\left\{-2\left(F^{\prime}\right)^{2} H\left[U^{\prime}\right]+\right. \\
\left.+F^{2} V^{\prime \prime \prime}-\frac{3}{2} F^{2} H\left[U^{\prime \prime \prime}\right]+2 F F^{\prime} V^{\prime \prime}-4 F F^{\prime} H\left[U^{\prime \prime}\right]\right\}+O\left(\epsilon^{3} U, \epsilon^{3} V\right)=0, \\
\Theta+\epsilon F H\left[\Theta^{\prime}\right]-\frac{1}{2} \epsilon^{2} F^{2} \Theta \Theta^{\prime \prime}+O\left(\epsilon^{3} \Theta\right) \\
=\epsilon B F+H\left[V^{\prime}\right]+U^{\prime}+\epsilon F\left\{H\left[U^{\prime \prime}\right]-V^{\prime \prime}\right\}-\frac{1}{2} \epsilon^{2} F^{2}\left\{H\left[V^{\prime \prime \prime}\right]+U^{\prime \prime \prime}\right\}+O\left(\epsilon^{3} U, \epsilon^{3} V\right), \\
\Theta-\Omega+\epsilon F H\left[\Theta^{\prime}+\Omega^{\prime}\right]-\frac{1}{2} \epsilon^{2} F^{2}\left\{\Theta^{\prime \prime}-\Omega^{\prime \prime}\right\}+O\left(\epsilon^{3} \Theta, \epsilon^{3} \Omega\right)=\epsilon D F, \\
\left\{\mathrm{I}-\frac{1}{2} \epsilon^{2}\left(F^{\prime}\right)^{2}+O\left(\epsilon^{4}\right)\right\}\left\{-H\left[\Theta^{\prime}+r \Omega^{\prime}\right]+\epsilon F\left(\Theta \Theta^{\prime \prime}-r \Omega^{\prime \prime}\right)+\frac{1}{2} \epsilon^{2} F^{2} H\left[\Theta^{\prime \prime \prime}+r \Omega^{\prime \prime \prime}\right]+\right. \\
\left.\quad+O\left(\epsilon^{3} \Theta, \epsilon^{3} \Omega\right)\right\}+\left\{\epsilon F^{\prime}+O\left(\epsilon^{3}\right)\right\}\left\{\Theta^{\prime}-r \Omega^{\prime}+\epsilon F H\left[\Theta^{\prime \prime}+r \Omega^{\prime \prime}\right]+O\left(\epsilon^{3} \Theta, \epsilon^{3} \Omega\right)\right\} \\
=2\left(\lambda / \lambda_{*}\right)^{2}\left[V-\epsilon(\mathrm{I}-\kappa) F^{\prime}-\epsilon(F U)^{\prime}+\epsilon^{2}\left\{-\frac{1}{2}\left(F^{\prime}\right)^{2} V+F F^{\prime} V^{\prime}-2 F F^{\prime} H\left[U^{\prime}\right]+\right.\right. \\
\left.\quad+\frac{1}{2} F^{2} V^{\prime \prime}-F^{2} H\left[U^{\prime \prime}\right]\right\}+O\left(\epsilon^{3} U, \epsilon^{3} V, \epsilon^{2} \kappa(\lambda / h) .\right.
\end{aligned}
$$

The argument $X$ is omitted throughout. If we anticipate the conclusions that $U, V, \Theta$ and $\Omega$ are $O(\epsilon)$ or smaller, and that $\kappa(\lambda / h)$ is $O\left(\epsilon^{2}\right)$ or smaller, then Equations $(62)-(65)$ contain explicitly the terms required to derive expansions of $U, V, \Theta$ and $\Omega$ to $O\left(\epsilon^{3}\right)$; that is, an approximation neglecting terms $O\left(\epsilon^{4}\right)$ compared with a leading term $O(\epsilon)$. Thus, for a smooth bed, the error may be only $\mathrm{I} \%$ for $\epsilon \approx 0.2$. Here terms to $O\left(\epsilon^{2}\right)$ only are derived explicitly. It is supposed that $B$ and $D$ are order unity in the balance, but later calculation with the adopted physical parameters shows that these geothermal flux terms contribute little to the $O(\epsilon)$ terms.

Consider the balance for $\lambda=O\left(\lambda_{*}\right)$, that is

$$
\omega=\left(\lambda / \lambda_{*}\right)=O(\mathbf{1}) .
$$


Alternative balances for smaller and larger $\omega$ have been obtained explicitly, and have been shown to be appropriate limits of the solution based on Equation (66). Let

$$
\left.\begin{array}{rl}
\kappa(\lambda / h) & =\gamma_{0}+\gamma_{1} \epsilon+\gamma_{2} \epsilon^{2}+\ldots, \\
U & =U_{0}+U_{1} \epsilon+U_{2} \epsilon^{2}+\ldots,
\end{array}\right\}
$$

with similar expressions for $V, \Theta$ and $\Omega$, and compare coefficients of $\epsilon^{0}, \epsilon^{1}, \epsilon^{2}$ in Equations $(62)-(65)$. The leading term of Equation (62) gives

$$
\gamma_{0}+H\left[U_{0}^{\prime}\right]=0,
$$

and by Equations (59) and (6o),

$$
\gamma_{0}=0, \quad U_{0}=0,
$$

since a non-zero constant $U_{0}$ leads to finite $\phi(Z)$ and $\psi(Z)$ as $Y \rightarrow \infty$, which violates Equation (49). By Equations $\left(6_{4}\right)$, (65) and $\left(6_{3}\right)$ in turn,

$$
\left.\begin{array}{rl}
\Omega_{0} & =\Theta_{0}, \\
-(\mathrm{I}+r) H\left[\Theta_{0}^{\prime}\right] & =2 \omega^{2} V_{0}, \\
\Theta_{0} & =H\left[V_{0}^{\prime}\right],
\end{array}\right\}
$$

and eliminating $\Theta_{0}$ using Equations $\left(5^{8}\right)$ and (59),

$$
V_{0}^{\prime \prime}-2 \omega^{2} V_{0} /(\mathrm{I}+r)=0 \text {. }
$$

Both solutions of Equation (7I) are unbounded at one limit, $X \rightarrow \pm \infty$, so

$$
\left.\begin{array}{l}
r_{0}=0, \\
\Theta_{0}=\Omega_{0}=0 .
\end{array}\right\}
$$

With Equations (69) and (72), the $\epsilon$ term of Equation (62) is analogous to Equation (68), giving

$$
\gamma_{1}=0, \quad U_{1}=0 .
$$

Now Equations $(64),\left(6_{3}\right)$ and $(65)$ give

$$
\left.\begin{array}{c}
\Omega_{1}=\Theta_{1}-D F, \\
\Theta_{1}=B F+H\left[V_{1}^{\prime}\right],
\end{array}\right\}
$$

where

$$
2 \omega^{2} / \mathrm{I}+r=\bar{\omega}^{2}=\left(\lambda / \bar{\lambda}_{*}\right)^{2},
$$

and

$$
\lambda_{*}^{2}=\lambda_{*}{ }^{2}(\mathrm{I}+r) / 2=2 \mu\left(k_{\mathrm{i}}+k_{\mathrm{b}}\right) C / L=0.0078 \mathrm{~m}^{2},
$$

for the adopted values $(r=\mathrm{I} .6) . \quad \lambda_{*}=0.088 \mathrm{~m}$ is the $\mathrm{Kamb}$ (1970) definition of the critical length scale. Both complementary functions of Equation (75) are unbounded at one limit, $X \rightarrow \pm \infty$, and $V_{1}$ is given by the bounded particular integral, simply a repeated quadrature once $F^{\prime}$ and $H\left[F^{\prime}\right]$ are known.

Now the $\epsilon^{2}$ term of Equation $(62)$ gives

$$
\gamma_{2}-F H\left[V_{1}^{\prime \prime}\right]=-H\left[U_{2}^{\prime}\right] \text {. }
$$

$H\left[U_{2}^{\prime}\right]$ and $H\left[V_{1}{ }^{\prime \prime}\right]$ are periodic, but the product $F H\left[V_{1}^{\prime \prime}\right]$ is in general the sum of a constant term $\Gamma$ and a periodic term $W(X)$, so the solution of Equation $(78)$ is

$$
\gamma_{2}=\Gamma, \quad U_{2}^{\prime}=-H[W] \text {. }
$$

This is demonstrated clearly by the subsequent sinusoidal bed calculation. Thus,

$$
\kappa \lambda / h=\Gamma \epsilon^{2}+O\left(\epsilon^{3}\right) .
$$


By Equation (75) $\Gamma$ contains a term $(\mathrm{I}-\kappa)$, so an explicit relationship between $\kappa, \lambda / h$ and $\epsilon^{2}$ is obtained for a given $F(X)$, and in turn we obtain expressions for $U_{\mathrm{s}}$ and $U_{\mathrm{b}} / U_{\mathrm{s}}$ where by Equations (27) and (69):

$$
U_{\mathrm{b}} / U_{\mathrm{s}}=\mathrm{I}-\kappa \text {. }
$$

The basal sliding velocity $U_{\mathrm{b}}$ is defined as the term of order unity in $V_{x}$ on $Y=0$. It will be shown in Figure 3 that the terms in $B$ and $D$ do not contribute to $\Gamma$, and hence do not affect $U_{\mathrm{s}}$ and $U_{\mathrm{b}}$, and furthermore that $\mathrm{o}<\kappa<\mathrm{I}$ as expected. It should be noted that the secondorder velocity term $U_{2}$ and the basal sliding velocity (of order unity) are determined together in an $O\left(\epsilon^{2}\right)$ balance.

Finally, to complete the second-order expansions, using Equations (64), (63) and (65),

$$
\begin{aligned}
& \Omega_{2}=\Theta_{2}+F H\left[\Theta_{1}{ }^{\prime}+\Omega_{1}{ }^{\prime}\right] \\
& \left.\Theta_{2}=H\left[V_{2}{ }^{\prime}\right]+U_{2}{ }^{\prime}-F V_{1}{ }^{\prime \prime}-F H\left[\Theta_{1}{ }^{\prime}\right]\right\} \text {, } \\
& V_{2}^{\prime \prime}-\bar{\omega}^{2} V_{2}=H\left[U_{2}^{\prime \prime}\right]-H\left[\left\{F V_{1}^{\prime \prime}\right\}^{\prime}\right]-H\left[\left\{F H\left[\Theta_{1}{ }^{\prime}\right]\right\}^{\prime}\right]+ \\
& +\{r /(\mathrm{I}+r)\} H\left[\left\{F H\left[\Theta_{1}{ }^{\prime}+\Omega_{1}{ }^{\prime}\right]\right\}^{\prime}\right]-\{\mathrm{I} /(\mathrm{I}+r)\}\left\{F\left(\Theta_{1}{ }^{\prime}-r \Omega_{1}{ }^{\prime}\right)\right\}^{\prime} .
\end{aligned}
$$

The latter has a particular integral for $V_{2}$ which is bounded and periodic. The product terms in Equation (82) allow constants to appear in $\Theta_{2}$ and $\Omega_{2}$. These represent second-order corrections to $T_{0}$ in the values of $T$ and $S$ on $Y-0$. Recall that $T_{0}$ was defined as the melting temperature corresponding to pressure $p_{0}$.

\section{Sinusoidal Bed profile}

The solution is now given explicitly for

$$
\left.\begin{array}{rl}
f(x) & =a \sin k x \\
\epsilon & =k a \\
\lambda & =\mathrm{r} / k \\
F(X) & =\sin X,
\end{array}\right\},
$$

with $F(X)=\cos X$ covered by the shift $X \rightarrow X+\pi / 2$. The contributions of the harmonics in a truncated Fourier series will be indicated. Only the Hilbert transforms (Equation (6o)) are required. We set

$$
B-\frac{r}{\mathrm{I}+r} D=A(\mathrm{I}-\kappa) \bar{\omega}^{2},
$$

as a result of which, Equation (75) becomes

$$
V_{\mathrm{I}}^{\prime \prime}-\bar{\omega}^{2} V_{\mathrm{I}}=-(\mathrm{I}-\kappa) \bar{\omega}^{2}(\cos X+A \sin X),
$$

with the bounded particular integral

$$
\Gamma_{\mathrm{I}}=\frac{(\mathrm{I}-\kappa) \bar{\omega}^{2}}{\bar{\omega}^{2}+\mathrm{I}}(\cos X+A \sin X) .
$$

Hence

$$
F H\left[I_{1}{ }^{\prime \prime}\right]=\frac{(\mathrm{I}-\kappa) \bar{\omega}^{2}}{2\left(\bar{\omega}^{2}+\mathrm{I}\right)}\{\mathrm{I}-\cos 2 X-A \sin 2 X\},
$$

so that

$$
\frac{\kappa \lambda}{h \epsilon^{2}}=\frac{(\mathrm{I}-\kappa) \bar{\omega}^{2}}{2\left(\bar{\omega}^{2}+\mathrm{I}\right)}+O(\epsilon),
$$

and

$$
U_{2}=\frac{(\mathrm{I}-\kappa) \bar{\omega}^{2}}{4\left(\bar{\omega}^{2}+\mathrm{I}\right)}\{\cos 2 X+A \sin 2 X\} .
$$


Thus $\kappa$, and hence $U_{\mathrm{b}}$ and $U_{\mathrm{s}}$, are independent of $A$ for $F(X)=\sin X$. This applies for any combination $a \sin X+b \cos X$ which becomes a sine function with a phase shift. If further harmonics are added, the cross products between different harmonics arising in $F H\left[V_{1}{ }^{\prime \prime}\right]$ contribute no constant terms, so $A$ cannot enter into Equation (89). The restriction of Equation (23) is met provided that $\kappa>O\left(\epsilon^{2}\right)$ for $\bar{\omega} \geqslant O(\mathrm{I})$, thus implying an upper bound to $\bar{\omega}$ (see Equation (9I)).

From Equations (28) and (8I) and the leading term of Equation (89),

$$
\begin{gathered}
\mathrm{o}<\frac{U_{\mathrm{b}}}{U_{\mathrm{s}}}=\mathrm{I}-\kappa=\frac{2\left(\bar{\omega}^{2}+\mathrm{I}\right) \lambda_{\sharp}}{\bar{\omega} h \epsilon^{2}} /\left\{\mathrm{I}+\frac{2\left(\bar{\omega}^{2}+\mathrm{I}\right) \lambda_{*}}{\bar{\omega} h \epsilon^{2}}\right\}<\mathrm{I}, \\
U_{\mathrm{b}}=\frac{\rho g \sin \alpha}{\mu} \frac{\bar{\omega}^{2}+\mathrm{I}}{\bar{\omega}} \frac{\lambda_{*} h}{\epsilon^{2}} .
\end{gathered}
$$

For a fixed ratio of $h / \epsilon^{2}$, the minimum value of $U_{\mathbf{b}}$ is given by:

$$
\left(U_{\mathrm{b}}\right)_{\min }=\frac{2 \bar{\lambda}_{*} \rho g \sin \alpha}{\mu \epsilon^{2}},
$$

and this occurs when $\lambda=\bar{\lambda}_{*}$. So, for $\lambda$ smaller or larger than $\bar{\lambda}_{*}$, the basal sliding velocity increases. Figure 2 shows the variation of $U_{\mathbf{b}}$ (in SI-units) with $\log \left(\lambda / \bar{\lambda}_{*}\right)$ for the physical

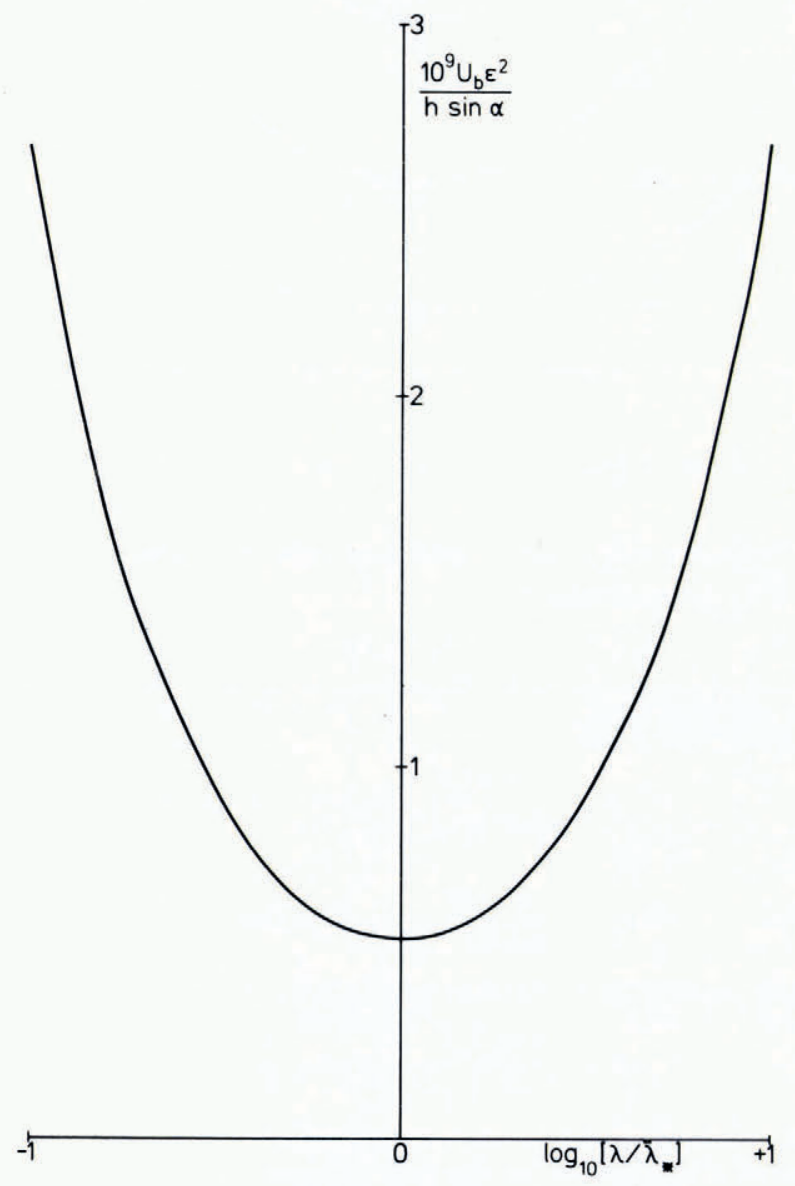

Fig. 2. Variation of basal sliding velocity $U_{1}$, with bed scale $\lambda$ (SI-units). 
data given earlier. At fixed values of $\lambda, U_{\mathbf{b}}$ increases with $h / \epsilon^{2}$. Figure 3 shows the variation of $U_{\mathrm{b}} / U_{\mathrm{s}}$ with $\log \left(\lambda / \bar{\lambda}_{*}\right)$ for different values of $h \epsilon^{2}$. For example, $h \epsilon^{2}=4$ applies for $h=100 \mathrm{~m}$ and $\epsilon=0.2$, while $h \epsilon^{2}=0.1$ applies for $h=10 \mathrm{~m}$ and $\epsilon=0.1$. However, the Newtonian approximation could have a serious effect on any quantitative predictions of $U_{\mathrm{b}}$ and $U_{\mathrm{s}}$, whereas the main purpose was to show that a flow field, which determines $U_{\mathrm{b}}$ and which is compatible with the gravity drive, pressure-melting, and regelation boundary conditions, exists. Alternatively, given $U_{\mathrm{b}}, U_{s}$ and $h$, Equations $(9 \mathrm{I})$ and $(92)$ determine the bed parameters $\epsilon$ and $\lambda$.

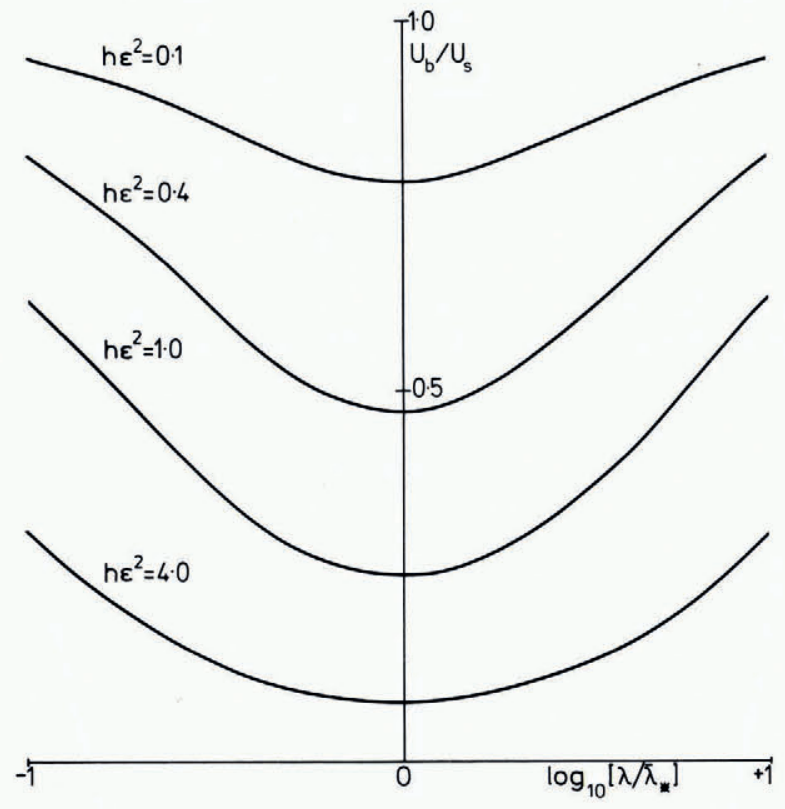

Fig. 3. Variation of the ratio of basal sliding zelocity to surface velocity with bed scale $\lambda$ for different zalues of h $\epsilon^{2}$ (SI-units).

Substitution of the above expressions indicates that the right-hand side of Equation $\left(8_{3}\right)$ is zero, and hence the bounded solution is

$$
V_{2}=0 \text {. }
$$

The temperature terms are now given by Equations (74) and (82). The velocity components of the bed line are, to second order,

$$
\left.\begin{array}{l}
U=\epsilon^{2} \frac{(\mathrm{I}-\kappa) \bar{\omega}^{2}}{4\left(\bar{\omega}^{2}+\mathrm{I}\right)}\{\cos 2 X+A \sin 2 X\}, \\
V=\epsilon \frac{(\mathrm{I}-\kappa) \bar{\omega}^{2}}{\bar{\omega}^{2}+\mathrm{I}}\{\cos X+A \sin X\} .
\end{array}\right\} .
$$

Using the estimates contained in Equations (37), (39), (85) and (89),

$$
A \approx \frac{0.54 \epsilon^{2}}{h \sin \alpha}\left(\frac{2 \bar{\omega}}{\bar{\omega}^{2}+1}\right) \leqslant \frac{0.54 \epsilon^{2}}{h \sin \alpha} .
$$

Taking the extreme values $h=10 \mathrm{~m}, \alpha=0.04$ and $\epsilon=0.2$, the maximum value of $A$ is 0.05 , so that the contribution made by the geothermal flux is small. We may recall that $B$ and hence $A$, are even smaller if $Q=0$, by Equation (36). 
The substitution of Equation (95) into Equations (50) and (5I) and the use of Equation (6I) gives, to second order,

$$
\left.\begin{array}{l}
\phi(z)=\frac{\epsilon \bar{\omega}^{2}(\mathrm{I}-\kappa)(\mathrm{I}-\mathrm{i} A)}{8\left(\bar{\omega}^{2}+\mathrm{I}\right)}\left\{4 \mathrm{e}^{\mathrm{i} z}-\epsilon \mathrm{i}^{2 \mathrm{i} z}\right\} \\
\psi(z)=\frac{\epsilon \bar{\omega}^{2}(\mathrm{I}-\kappa)(\mathrm{I}-\mathrm{i} A)}{8\left(\bar{\omega}^{2}+\mathrm{I}\right)}\left\{4 \mathrm{e}^{\mathrm{i} z}+\epsilon \mathrm{i}^{2 \mathrm{i} z}\right\}-z \phi^{\prime}(z)
\end{array}\right\},
$$

which determine the velocity and stress fields through Equations (43)-(46) and Equations (27)-(30), and which show the exponential decay in $r$. In particular

$$
P=\frac{\epsilon \bar{\omega}^{2}(\mathrm{I}-\kappa)}{2\left(\bar{\omega}^{2}+\mathrm{I}\right)}\left\{2 \mathrm{e}^{-Y}(\cos X+A \sin X)+\epsilon \mathrm{e}^{-2 Y}(\sin 2 X-A \cos 2 X)\right\} .
$$

We may note that

$$
\frac{2 \mu U_{\mathrm{s}}}{\lambda} \frac{\bar{\omega}^{2}(\mathrm{I}-\kappa)}{2\left(\bar{\omega}^{2}+\mathrm{I}\right)}=\frac{\rho g \sin \alpha h}{\epsilon^{2}}
$$

and hence the pressure field becomes

$$
p=p_{\mathrm{a}}+\rho g h \cos \alpha\left\{\mathrm{I}-\frac{\lambda}{h} Y+\frac{\tan \alpha}{\epsilon} \mathrm{e}^{-Y}\left[2 \cos X+\epsilon \mathrm{e}^{-Y} \sin 2 X\right]\right\},
$$

when $A$ is neglected.

On the bed surface

$$
p_{\mathrm{b}}=p_{\mathrm{a}}+\rho g h \cos \alpha\left\{\mathrm{I}+\frac{2 \tan \alpha}{\epsilon}[\cos X+O(\epsilon)]+O(\epsilon)\right\},
$$

and since the present solution is valid only when the ice boundary coincides with the bed surface (that is, no cavitation occurs) we require $p_{\mathrm{b}} \geqslant 0$ which implies that

$$
\tan \alpha \leqslant \frac{\epsilon}{2}\left(1+\frac{p_{\mathrm{a}}}{\rho g h \cos \alpha}\right) .
$$

The factor $\left(\mathrm{I}+p_{\mathrm{a}} /(\rho g h \cos \alpha)\right)$ is approximately equal to one for $h \geqslant 100 \mathrm{~m}$, and even for a thin glacier $h=10 \mathrm{~m}$ (Equation ( 102$)$ ) only allows $\alpha \leqslant \epsilon$. So, cavitation is predicted for moderate bed inclination. Kamb (1970) infers an instability criterion of $\alpha>\epsilon$ from an argument involving the value of the absolute bed slope, but this appears to be invalid unless the resisting "pressure drag" is eliminated from the lee flanks of the undulations by "total cavitation" there. While Equation ( 102 ) shows that cavitation does occur for $\alpha>\epsilon$ (for a sinusoidal bed on the Newtonian approximation), it is still reasonable to expect that pressure over the contact section of lee flanks will be sufficient to provide the necessary drag, but a full solution which incorporates cavitation is needed in order to confirm this.

\section{Concluding remarks}

We now see that slow viscous plane flow over an inclined wavy bed has a bounded solution consistent, at least to the order of $\epsilon^{2}$, with the perturbation on the laminar flow decaying rapidly with height. This is in contrast to calculations of half-plane flow which are subjected to the classical zero velocity condition at the bed-line. The inclusion of gravity in the momentum balance provides the driving mechanism and also defines the mean drag directly. Thus, the unbounded terms arising from the artificial driving shear stress of Nye do not arise. The terms in $\epsilon$ agree with the first-order expansions of Nye and Kamb, but, whereas Nye derives a relationship between the mean drag and the basal sliding velocity from the firstorder solution, the present solution shows how the surface and basal velocities are governed 
by the second-order balance and are given directly. It is shown that a uniform geothermal flux does not influence the basal-sliding velocity, and makes only a small contribution to the flow field.

The application of boundary conditions to the bed surface instead of the half-plane boundary allows direct expansions beyond the first power in $\epsilon$. The balance equations have been given up to terms in $\epsilon^{3}$ so that a solution for moderate slopes $(\epsilon \sim 0.2)$ could be obtained. In the dimensionless expansion analysis, coefficients should remain of order unity if the higher derivatives of the bed profile are also small. However, the complex variable method of conformal mapping offers a direct approach to the problem of flow over a hump of finite slope.

\section{Acknowledgement}

This investigation was pursued in connection with a Natural Environment Research Council Grant GR 3/268o "Flow of glaciers over deformable materials" which I hold jointly with Dr G. S. Boulton of the School of Environmental Sciences, University of East Anglia.

$M S$. received 20 September 1975 and in revised form 8 January 1976

\section{REFERENCES}

Erdélyi, A., ed. 1954. Tables of integral transforms. Vol. 2. Based, in part, on notes left by Harry Bateman. New York, etc., McGraw-Hill Book Co., Inc.

Kamb, W. B. 1970. Sliding motion of glaciers: theory and observation. Reviews of Geophysics and Space Physics, Vol. 8, No. 4, p. 673-728.

Langlois, W. E. I 964 . Slow viscous flow. New York, Macmillan.

Lliboutry, L. A. 1968 . General theory of subglacial cavitation and sliding of temperate glaciers. Journal of Glaciology, Vol. 7, No. 49, p. 2 1-58.

Muskhelishvili, N. I. 1954. Nekotoryye osnovnyye zadachi matematicheskoy teorii uprugosti; osnovnyye uravneniya, ploskaya teoriya uprugosti, krucheniye $i$ izgib. Izdaniye 4. Moscow, Izdatel'stvo Akademii Nauk SSSR. [English translation: Some basic problems of the mathematical theory of elasticity. Translated from the Russian by $\mathcal{J}$. R. M. Radok. Groningen, Noordhoff, 1963.]

Nye, J. F. 1969. A calculation on the sliding of ice over a wavy surface using a Newtonian viscous approximation. Proceedings of the Royal Society of London, Ser. A, Vol. 31 1 , No. 1506, p. 445-67.

Nye, J. F. 1970. Glacier sliding without cavitation in a linear viscous approximation. Proceedings of the Royal Society of London, Ser. A, Vol. 315 , No. 1522 , p. $381-403$.

Paterson, W. S. B. 1969 . The physics of glaciers. Oxford, etc., Pergamon Press. (The Commonwealth and International Library. Geophysics Division.)

Weertman, J. 1957. On the sliding of glaciers. Journal of Glaciology, Vol. 3, No. 21, p. 33-38.

Weertman, J. I964. The theory of glacier sliding. Fournal of Glaciology, Vol. 5, No. 39, p. 287-303.

Weertman, J. 1971. In defense of a simple model of glacier sliding. Journal of Geophysical Research, Vol. 76, No. 26, p. $6485-87$. 